\title{
Expected degree of RNA secondary structure networks
}

\author{
Peter Clote
}

\begin{abstract}
Consider the network of all secondary structures of a given RNA sequence, where nodes are connected when the corresponding structures have base pair distance one. The expected degree of the network is the average number of neighbors, where average may be computed with respect to the either the uniform or Boltzmann probability. Here we describe the first algorithm, RNAexpNumNbors, that can compute the expected number of neighbors, or expected network degree, of an input sequence. For RNA sequences from the Rfam database, the expected degree is significantly less than the CMFE structure, defined to have minimum free energy over all structures consistent with the Rfam consensus structure. The expected degree of structural RNAs, such as purine riboswitches, paradoxically appears to be smaller than that of random RNA, yet the difference between the degree of the MFE structure and the expected degree is larger than that of random RNA. Expected degree does not seem to correlate with standard structural diversity measures of RNA, such as positional entropy, ensemble defect, etc. The program RNAexpNumNbors is written in $\mathrm{C}$, runs in cubic time and quadratic space, and is publicly available at http://bioinformatics.bc.edu/clotelab/RNAexpNumNbors
\end{abstract}

\section{Introduction}

Examples of small-world phenomena abound in the physical sciences. In [26, the neural connections of $C$. elegans were shown to have both small mean path length between nodes and to have large clique-like clusters, both hallmarks of small-world networks. In 23, the gene co-expression network of S. cerevisiae was shown to have a scale-free, small-world architecture, where genes are linked in the network if they are co-expressed. The network of metabolic pathways [24, 16] and of folding kinetics for the protein villin [2] were shown as well to have small-world properties, which collectively suggests a topological robustness that appears to be intrinsic in biological processes.

In [27, Wuchty showed that the network of secondary structures of E. coli phe-tRNA, with accession number RF6280 20], is small-world, by using the program RNAsubopt 28] to analyze the ensemble of low energy secondary structures of phe-tRNA, having free energy approximately within $8.5 \mathrm{kcal} / \mathrm{mol}(14 \mathrm{kT})$ of the minimum free energy. In this network, two nodes $s, t$ (secondary structures) are linked by an edge if $t$ can be obtained from $s$ by removing or adding a single base pair, or obtained by a shift move. A shift move, depicted in Figure 1 of [27, allows one to move one end of a base pair without moving the other.

Small-world networks satisfy a connectivity property, where the average shortest path distance (geodesic distance) is small relative to the network size - typically logarithmic in network size. In the case of RNA secondary structures, this property trivially holds, since the number of secondary structures is generally exponential in the RNA sequence size $n$ [21, while the base pair distance between any two structures (and so path length) is at most $n$.

Wuchty showed as well that cluster sizes of the low energy ensemble of secondary structures of phe-tRNA are larger than that of (Erdös-Renyi) random graphs, and that clustering coefficient $C(\nu)$ for phe-tRNA is inversely proportional to node degree $k_{\nu}$ of node $\nu$; i.e. $C(\nu) \sim \frac{1}{k_{\nu}}$. Here, for network node $\nu$, the clustering coefficient $C(\nu)$ is defined as the fraction of pairs of neighbors of $\nu$ which are connected by a network edge; i.e. $C(\nu)=\frac{2 \cdot n}{k_{\nu} \cdot\left(k_{\nu}+1\right)}$, where $n$ is the number of pairs of neighbors of $\nu$ that are connected by a network edge.

Motivated originally by issues concerning the kinetics of RNA folding, in this paper we study the average network degree, i.e. the expected number of neighbors of a secondary structure for a given RNA sequence. In contrast to the work of Wuchty [27, we consider a neighbor of the secondary structure $s$ to be any structure obtained by adding or deleting a single base pair from $s$, but not obtained by a shift move. We describe the first algorithm, RNAexpNumNbors, that can compute the expected number of neighbors for a given RNA 
sequence, where expectation can be taken either with respect to the uniform or the Boltzmann probability. Using dynamic programming, the $\mathrm{C}$ program RNAexpNumNbors runs in cubic time and quadratic space with respect to input sequence length.

The plan of the paper is as follows. To make basic notions absolutely clear, Section 2 presents an illustrative example of how to manually compute the expected network degree for a toy $8 \mathrm{nt}$ RNA sequence. Section 3 shows the inadequacy of this exhaustive method, hence the need for an efficient program such as RNAexpNumNbors. In this section, we apply RNAexpNumNbors to nine noncoding RNA families from the Rfam database [10, and determine the Pearson correlation between expected network degree and various RNA structural measures. Definitions for these measures are given in the Appendix, and the correlations are displayed in Tables 1 to 5 . Section 4 summarizes our main contributions and poses some open questions. The recursions for our algorithm, RNAexpNumNbors, are described in Section 5 , and the detailed derivation for these recursions are given in the Appendix.

\section{Preliminaries}

A secondary structure for an RNA nucleotide sequence $\mathbf{a}=a_{1}, \ldots, a_{n}$ is a set $s$ of Watson-Crick or wobble base pairs $(i, j)$, containing neither base triples nor pseudoknots. The number of base pairs in $s$ is denoted by $|s|$. A secondary structure $s$ is compatible with a if for every base pair $(i, j)$ in $s$, the pair $\left(a_{i}, a_{j}\right)$ is contained in the set of six canonical (Watson-Crick and wobble) base pairs. Throughout this paper, by structure, we mean a secondary structure which is compatible with an arbitrary, but fixed RNA sequence $\mathbf{a}$.

If $s, t$ are secondary structures of a, then the base pair distance, $d_{B P}(s, t)$, is defined as $|s-t|+|t-s|$, i.e. the number of base pairs belonging to $s$ but not $t$, or vice versa. Structures $s, t$ are said to be neighbors, if their base pair distance is 1 , and to be $k$-neighbors if $d_{B P}(S, T)=k$.

Given an RNA sequence $\mathbf{a}=a_{1}, \ldots, a_{n}$, the expected number $\langle N(\mathbf{a})\rangle$ of neighbors for $\mathbf{a}$ is defined by

$$
\langle N(\mathbf{a})\rangle=\sum_{s} P(s) \cdot N(s)=\sum_{s} \frac{\exp (-E(s) / R T)}{Z} \cdot N(s)
$$

where the sum is taken over all secondary structures $s$ of the input sequence $\mathbf{a}$, where $P(s)$ is the probability of structure $s, N(s)$ is the number of neighbors of $s, E(s)$ is the energy of $s, R=0.001987 \mathrm{kcal} \mathrm{K}^{-1} \mathrm{~mol}^{-1}$ and $T=310.15 \mathrm{~K}$. At times we may write $N_{s}$ in place of $N(s)$, especially in Section 5 and the Appendix. Often the RNA sequence $\mathbf{a}$ is clear from the context and so omitted, and we may correspondingly designate the expected number of neighbors by $\langle N\rangle$.

In this paper, we describe novel, efficient (cubic time, quadratic space) algorithms to compute the expected number of neighbors, with respect to three energy models. Model A assigns an energy of 0 to each structure. It follows that the partition function $Z=\sum_{s} \exp (-E(s) / R T)$ is simply the number of structures, and so the probability $P(s)=\frac{\exp (-E(s) / R T)}{Z}$ is the uniform probability. Model $\mathrm{B}$, first proposed by Nussinov and Jacobson [15], assigns an energy of -1 to each base pair, so the energy of a structure having $k$ base pairs is $-k$. Model C, commonly known as the Turner energy model [13, 29, assigns negative, stabilizing free energies to stacked base pairs, and positive, destabilizing free energies to hairpin loops, bulges, internal loops and multiloops. The energy parameters are derived from UV absorption (optical melting) experiments, except for a multiloop affine energy approximation. In our current software RNAexpNumNbors, we employ the Turner 1999 energy parameters [13, 29, 22 without dangles. Accounting for dangles, or single-stranded, stacked nucleotides, would add considerable complexity to our dynamic programming algorithm; indeed, at present, it is unclear how this might even be done.

Although the Turner energy model is the only physically realistic model, involving enthalpic and entropic considerations, our dynamic programming method is complicated and more easily explained by first addressing model $\mathrm{A}$, then $\mathrm{B}$, then $\mathrm{C}$. To illustrate the definition of expected number of neighbors, given in equation (1), consider the 8 nt RNA sequence ACGUACGU, all of whose secondary structures can be generated by the program RNAsubopt [28] - see Figure 1.

Due to steric constraints, by definition each hairpin loop is required to have at least three unpaired bases. It follows that the first structure in Figure 1 has 2 neighbors, i.e. either the empty structure, obtained by removing base pair $(1,8)$, or the second structure, obtained by adding the base pair $(2,7)$. The second structure has 2 neighbors, obtained by removing base pair $(1,8)$ or $(2,7)$. The third structure has only one 


$\begin{array}{ll}(\ldots \ldots) & 4.40 \mathrm{kcal} / \mathrm{mol} \\ ((\ldots)) & 1.90 \mathrm{kcal} / \mathrm{mol} \\ \ldots(\ldots) & 5.70 \mathrm{kcal} / \mathrm{mol} \\ .(\ldots) & 3.60 \mathrm{kcal} / \mathrm{mol} \\ \ldots \ldots . & 0.00 \mathrm{kcal} / \mathrm{mol}\end{array}$

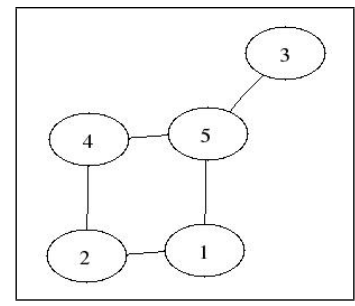

Figure 1: (Left) All possible secondary structures of the 8-mer ACGUACGU with corresponding free energies in kcal/mol, computed by RNAeval from the Vienna RNA Package [1] using the Turner 1999 energy model without dangles [13, 29]. (Right) Graph representation of neighborhood network, where nodes 1,2,3,4,5 respectively represent the secondary structures $(\ldots \ldots) ;((\ldots).) ; \ldots(\ldots) ; .(\ldots) . \ldots \ldots$. . . . . . . . . . . expected number of neighbors corresponds to the expected degree in the graph with respect to the uniform probability. In computing the expectation with respect to the Boltzmann probability, each node is weighted by its Boltzmann factor divided by the partition function.

neighbor, obtained by removing base pair $(3,8)$, while the fourth structure has two neighbors, obtained by either removing base pair $(2,7)$ or adding base pair $(1,8)$. In contrast the fifth structure, which is empty, has 3 neighbors, obtained by adding either base pair $(1,8),(2,7)$ or $(3,8)$. If the energy of each structure is 0 , as in Model A, then equation (1) yields $\frac{2+2+1+2+3}{5}=2$; i.e. the uniform expected number of neighbors is 2.

In Model B, usually called the Nussinov energy model, the partition function $Z$ is the sum of the Boltzmann factors $\frac{\exp (-E(s) / R T}{Z}$, hence

$$
\begin{aligned}
Z= & \exp (1 / R T)+\exp (2 / R T)+\exp (1 / R T)+ \\
& \exp (1 / R T)+\exp (0 / R T)=41.8611
\end{aligned}
$$

Thus, with respect to the Nussinov energy model, the Boltzmann expected number of neighbors is

$$
\begin{aligned}
\langle N\rangle= & \frac{\exp (1 / R T)}{Z} \cdot 2+\frac{\exp (2 / R T)}{Z} \cdot 2+\frac{\exp (1 / R T)}{Z} \cdot 1+ \\
& \frac{\exp (2 / R T)}{Z} \cdot 2+\frac{\exp (0 / R T)}{Z} \cdot 3=\frac{79.656}{41.861}=1.903 .
\end{aligned}
$$

In the Turner energy model, the free energies of the five structures are $4.4,1.9,5.7,3.6$ and $0.0 \mathrm{kcal} / \mathrm{mol}$, reflecting the fact that the minimum free energy structure for this toy 8-mer is the empty structure. The partition function is

$$
\begin{aligned}
Z= & \exp (-4.4 / R T)+\exp (-1.9 / R T)+\exp (-5.7 / R T)+ \\
& \exp (-3.6 / R T)+\exp (-0 / R T)=1.049626
\end{aligned}
$$

Thus, with respect to the Turner energy model, the Boltzmann expected number of neighbors is

$$
\begin{aligned}
\langle N\rangle= & \frac{\exp (-4.4 / R T) \cdot 2}{Z}+\frac{\exp (-1.9 / R T) \cdot 2}{Z}+\frac{\exp (-5.7 / R T) \cdot 1}{Z}+ \\
& \frac{\exp (-3.6 / R T) \cdot 2}{Z}+\frac{\exp (-0.0 / R T) \cdot 3}{Z}=\frac{3.0992}{1.0496}=2.9526 .
\end{aligned}
$$

In the sequel, the phrase Boltzmann expected number of neighbors will mean that the expected value is computed with respect to Boltzmann probability using the Turner 1999 energy model without dangles - i.e. Model C. A future version of RNAexpNumNbors will alternatively support the Turner 2000 parameters.

\section{Results}

It is straightforward, to automate the previous manual computations, and thus determine the expected number of neighbors for a given RNA sequence by exhaustively listing all secondary structures and their free energies with the program RNAsubopt [28]. This approach is only posssible for a sufficiently small RNA sequence, 

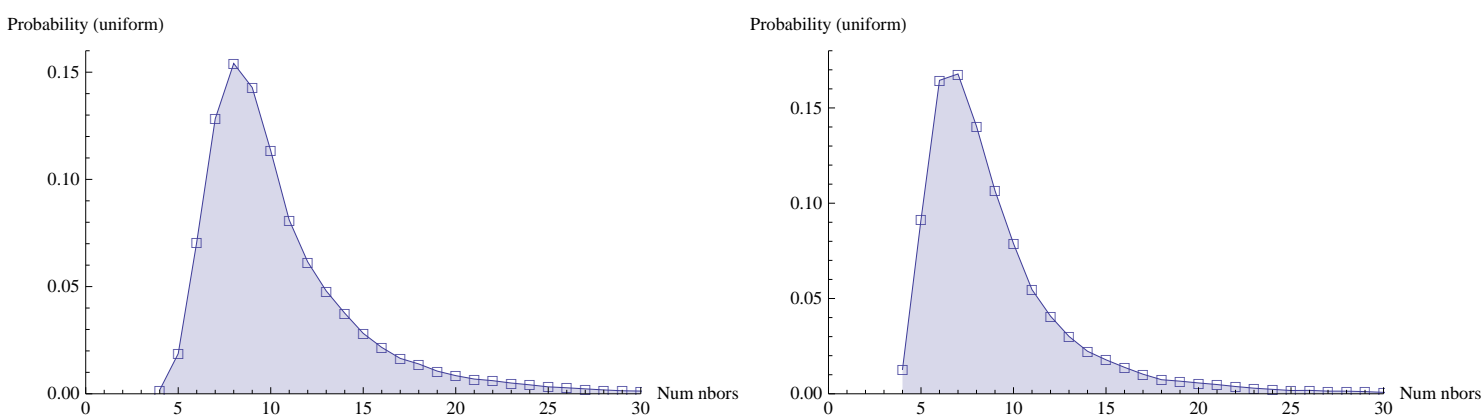

Figure 2: (Left) Relative frequency of the number of structures for the 32 nt selenocysteine insertion sequence (SECIS) element fruA, obtained by by exhaustive generation of all secondary structures. The number of neighbors is given on the $x$-axis, while the proportion of structures having a given number of neighbors is given on the $y$-axis. The expected number of neighbors with respect to the uniform distribution is 10.657 with standard deviation 4.777, while that for the Boltzmann probability is 10.001 with standard deviation 0.058. (Right) Similar analysis for a $27 \mathrm{nt}$ bistable switch. The expected number of neighbors with respect to the uniform distribution is 9.14 with with standard deviation 4.56, while that for the Boltzmann probability is 11.92 with standard deviation 0.549 .

since the number of secondary structures is exponential in the sequence length [21]. Binning the output of RNAsubopt according to the number of neighbors of each secondary structure, we can determine the relative frequency of structures of the $32 \mathrm{nt}$ selenocysteine insertion sequence (SECIS) element fruA with sequence CCUCGAGGGG AACCCGAAAG GGACCCGAGA GG and the 27 nt bistable switch with sequence CUUAUGAGGG UACUCAUAAG AGUAUCC and two meta-stable structures ....... (((((((( (.. $)))))))$ having $-10.30 \mathrm{kcal} / \mathrm{mol}$ and $((((((((\ldots)))))))) \ldots \ldots$ having $-9.90 \mathrm{kcal} / \mathrm{mol}$.

Figure 2 displays the relative frequency with respect to the uniform probability, that structures for fruA (left panel) resp. the bistable switch (right panel) have a given number of neighbors. In particular, 110,124 of the 971,299 secondary structures of fruA $(11.3 \%)$ have exactly 10 neighbors. The analogous computation with respect to the Boltzmann probability indicates that $99.8 \%$ of the structures have exactly 10 neighbors - of course, this means that essentially all of the low energy structures have 10 neighbors. The expected number of neighbors for fruA with respect to the uniform distribution is 10.657 (stdev 4.777), while that for the Boltzmann probability is 10.001 (stdev 0.058). For the 27 nt bistable switch, 30,609 of the 186,105 secondary structures (16.4\%) have exactly 6 neighbors, with respect to the uniform probability, while $88.36 \%$ of the structures have 12 neighbors with respect to the Boltzmann probability. The MFE structure having $-10.30 \mathrm{kcal} / \mathrm{mol}$ has 12 neighbors, while the meta-stable structure having $-9.90 \mathrm{kcal} / \mathrm{mol}$ has 11 neighbors.

For larger sequences, one can use RNAsubopt to sample those structures, whose free energy lies within a user-specified bound of that of the MFE structure. For the $161 \mathrm{nt}$ xanthine phosphoribosyltransferase (XPT) riboswitch, depicted in Figure 1 of [19], we used RNAsubopt to sample 8212 structures having free energy within $5 \mathrm{kcal} / \mathrm{mol}$ of the MFE. By computing the relative frequency that sampled structures have exactly $k$ neighbors, we obtain the density plot given in the left panel of Figure 3 , which yields the estimated mean 138.848 (stdev 45.457), compared with the correct value of 153.179 obtained with RNAexpNumNbors. By increasing the free energy bound of $5 \mathrm{kcal} / \mathrm{mol}$ to $10 \mathrm{kcal} / \mathrm{mol}$, one could obtain an improved graph and somewhat more accurate estimate of the mean; however, this comes at a severe computational cost, since the number of structures grows exponentially in the free energy bound. (Compare the left panel of Figure 3 with that of Figure 4 from [27.)

We wrote a program in $\mathrm{C}$ to count the number of secondary structures for an input RNA sequence and output a user-specified number of structures, sampled with respect to the uniform distribution. In this fashion, we generated 8000 structures from the ensemble of all structures of the XPT riboswitch, and determined an estimate of 70.326 with standard deviation of 16.249 for the uniform expected number of neighbors of XPT riboswitch. The exact value is 61.040, as determined by RNAexpNumNbors. The right panel of Figure 3 depicts the graph of uniform distribution of the number of neighbors for XPT.

It follows from this illustrative example that there is no current method, apart from the algorithm RNAexpNumNbors of this paper, which can accurately compute the expected number of neighbors for a given 

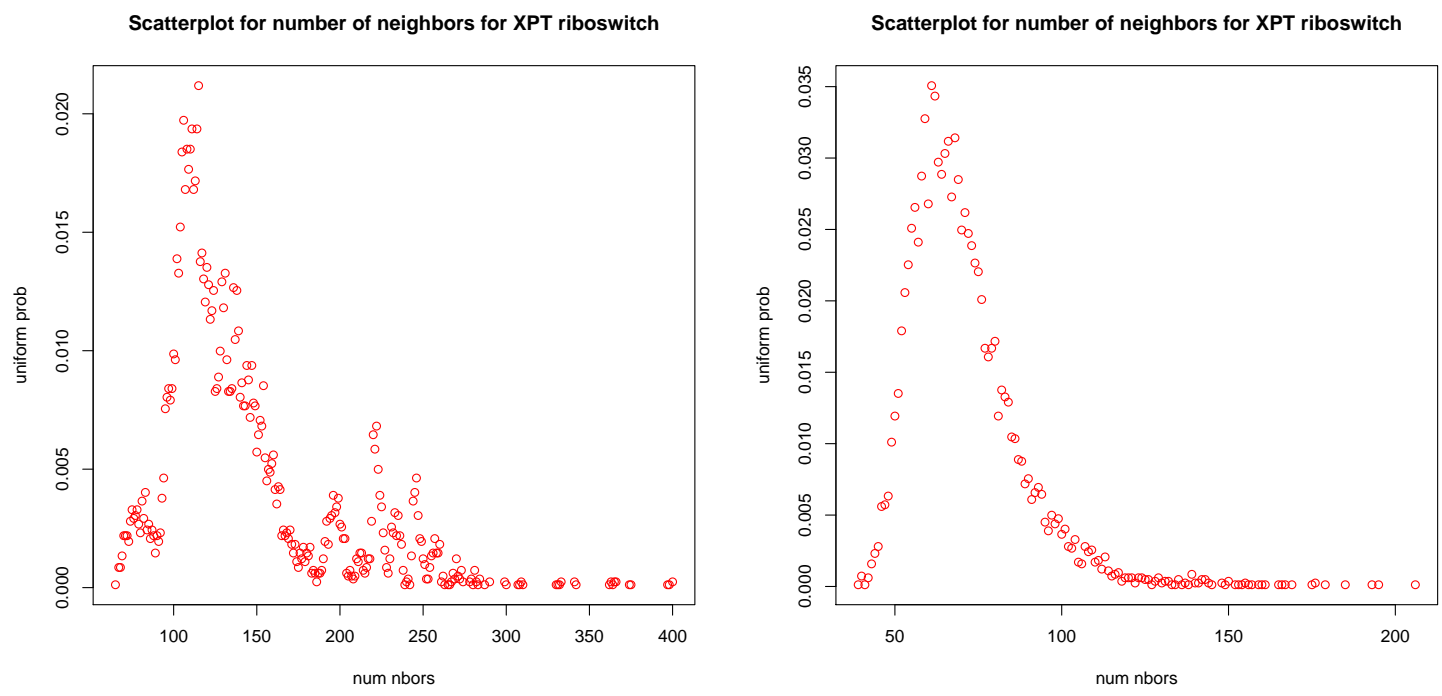

Figure 3: (Left) Relative frequency of the number of structures for the $161 \mathrm{nt}$ xanthine phosphoribosyltransferase (XPT) riboswitch, estimated by using RNAsubopt to sample 8212 structures having free energy within $5 \mathrm{kcal} / \mathrm{mol}$ of the MFE. Structures were binned according to number of neighbors, yielding a mean of 138.848 with standard deviation of 45.457, compared with the exact value of 153.179 obtained with RNAexpNumNbors. (Right) Analogous graph, where 8000 structures were uniformly sampled from the ensemble of all structures for the XPT riboswitch. This data yields an estimate of $70.326 \pm 16.249$ for the uniform expected number of neighbors of XPT, compared with the exact value of 61.040 obtained with RNAexpNumNbors.

RNA sequence. The dynamic programming recursions are described in Section 5, while full details of the derivation of the recursions is given in the Appendix.

\section{Analysis of selected Rfam families}

In this section, we apply RNAexpNumNbors to compute the expected number of neighbors, both with respect to the uniform and Boltzmann probability, for noncoding RNA from the Rfam 11.0 database [10. The nine Rfam families are 5S ribosomal RNA (RF00001), U2 spliceosomal RNA (RF00004), transfer RNA (RF00005), type III hammerhead ribozyme (RF00008), Selenocysteine insertion sequence 1 (RF00031), small nucleolar RNA (RF00045), purine riboswitch (RF00167), HIV primer binding site (RF00375), molybdenum cofactor riboswitch (RF01055).

Since it is clear that longer RNA sequences in general have a larger number of neighbors, these values should be normalized for comparative purposes. The left panel of Figure 4 depicts the expected number of neighbors, normalized by dividing by sequence length, for homopolymers of length 10 to 1000 . In this context, a homopolymer is a sequence, where any two positions $i<j$ can form a base pair, as long as $j-i \geq 4$, which ensures a minimum of at least three unpaired bases in each hairpin loop. Figure 4 clearly indicates that the normalized expected number of neighbors is asymptotically a constant in the homopolymer case, with asymptotic value $\approx 0.4724$. The center [resp. right] panels of Figure 4 plot the uniform [resp. normalized uniform] expected number of neighbors pooled from all sequences in the seed alignments of the nine Rfam families: RF00001, RF00004, RF00005, RF00008, RF00031, RF00045, RF00167, RF00375, RF01055. Sequence lengths in this pooled set range from $40 \mathrm{nt}$ to $225 \mathrm{nt}$, with a mean of $104.21 \pm 39.96$. No visible pattern emerges in the center panel, corresponding to unnormalized values. In normalizing by dividing the expected number of neighbors by sequence length, the values appear to be normally distributed with a mean of $0.3697 \pm 0.0091$. These arguments justify our normalized expected number of neighbors, when comparing RNAs of different lengths from different Rfam families.

Table 1 presents the averages, taken over all sequences in the seed alignment of each of nine selected families in the Rfam 11.0 database [10, of the expected number of neighbors with respect to both the 

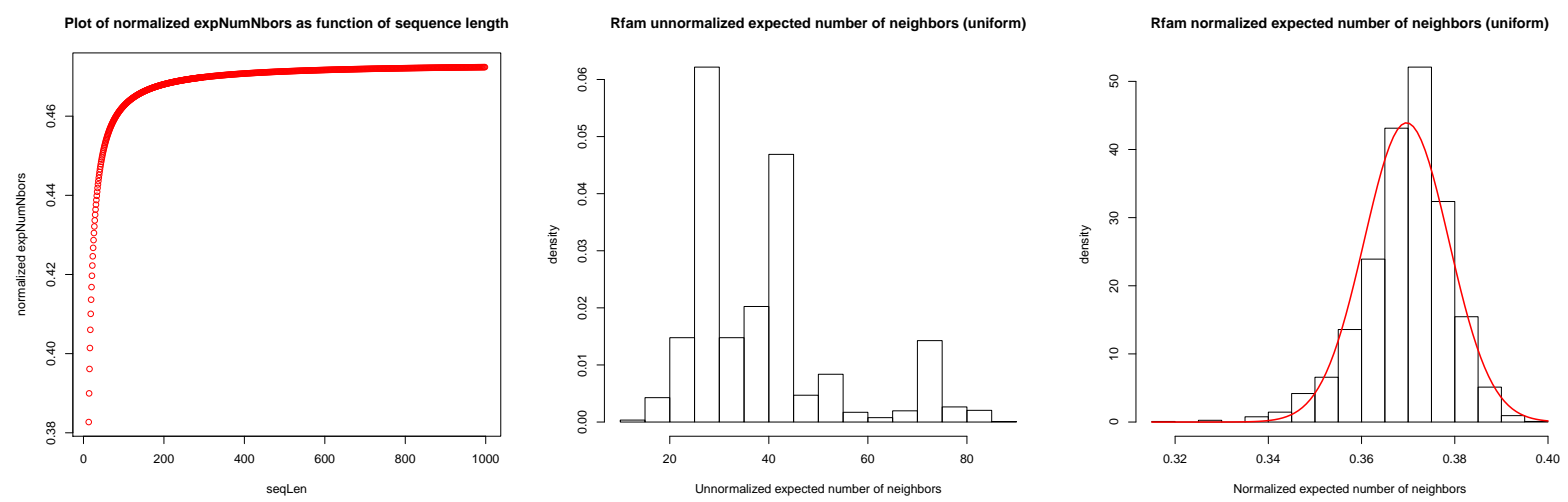

Figure 4: (Left) Plot of the normalized expected number of neighbors as a function of sequence length, for homopolymers of length 1 to $1000 \mathrm{nt}$, obtained by dividing the expected number of neighbors by sequence length. Apparent asymptotic value is $\approx 0.4724$. (Center) Relative frequency of the expected number of neighbors pooled from all sequences in seed alignments from nine Rfam families (see text). (Right) Relative frequency of the normalized expected number of neighbors pooled from all sequences in seed alignments from nine Rfam families, with a fitted normal distribution.

uniform probability and to the Boltzmann probability. Though small, the different values for the expected number of neighbors are statistically significant. For instance, the $p$-value is $2.00723 \cdot 10^{-46}$, for the 2 -tailed T-test of equality for the average (uniform) normalized number of neighbors for sequences from the seed alignment of RF00001 (712 5S rRNA sequences) and RF00005 (960 tRNA sequences).

The normalized expected number of neighbors (Boltzmann probability) appears to be completely uncorrelated with the normalized expected number of neighbors (uniform probability) - taken over the pooled data from nine Rfam families, the Pearson correlation is only 0.028191, as shown in Table2. This table considers as well the number of neighbors of the minimum free energy (MFE) structure and the the constrained minimum free energy (CMFE), where for the latter, we used RNAfold -C from the Vienna RNA Package 11. to compute the structure having minimum free energy among all structures that are compatible with the Rfam consensus structure. By this, we mean that the CMFE structure $s$ obtained by RNAfold $-C$ does not conflict with the constraints; if position $k$ is constrained to be unpaired, then position $k$ must be unpaired in $s$, and if $(x, y)$ is constrained to be a base pair, then for every base pair $(i, j) \in s$, we have that if $\{i, j\}$ and $\{x, y\}$ have non-empty intersection, then $i=x$ and $j=y$, and that it is not the case that $x<i<y<j$ or $i<x<j<y$. Note that RNAfold $-\mathrm{C}$ does not require that the base pair $(x, y)$ from the constraint belong to $s$, but only that $s$ not conflict with the constrained base pair.

As far as we can determine, the expected number of neighbors, equivalent to network degree, seems to be orthologous to other measures. In particular, there appears to be no relation between length-normalized Boltzmann expected number of neighbors (EXPB), length-normalized uniform expected number of neighbors (EXPU), GC-content, minimum free energy, sequence length, positional entropy [12, expected number of base pairs [25, ensemble defect 4], expected base pair distance [9], etc. Table 3 shows the absence of correlation between EXPB and various structural diversity measures (data for other measures not shown). See the Appendix for definitions of positional entropy, expected number of base pairs, ensemble defect, expected base pair distance.

Table 4 presents correlations between the expected number of neighbors and other measures, defined as follows. E: minimum free energy; MFE-EXPB: (number of neighbors of MFE structure minus the Boltzmann expected number of neighbors) divided by sequence length - i.e. length-normalized; EXPB: Boltzmann expected number of neighbors, divided by sequence length; MFE-EXPU: (number of neighbors of MFE structure minus the uniform expected number of neighbors) divided by sequence length; MFE: number of neighbors of the MFE structure divided by sequence length; SeqLen: sequence length.

Some correlations are obvious; e.g. corr $(\mathrm{E}, \mathrm{SeqLen})=-0.8621$ indicates that as sequence length increases, the minimum free energy decreases. The correlation of 0.8724 between MFE-EXPB and MFE-EXPU is significant and surprising, since there is essentially no correlation between EXPB and EXPU, as shown in 
Table 2 The positive correlation of 0.8721 between MFE-EXPB and MFE, and of 0.999436 between MFEEXPU and MFE seems surprising. However, since EXPB [resp. EXPU] values of members of a given Rfam family appear to be close to the family average (see Table 1), taken together this suggests that the value of MFE essentially defines the values of MFE-EXPB [resp. MFE-EXPU]. Finally, the correlation between MFE and EXPB is likely due to the fact that MFE-EXPB is small, in general, as shown in Table 5 .

Finally, Table 5 compares the number of neighbors of the MFE and CMFE structures with the expected number. In this table, MFE [resp. CMFE] stands for the length-normalized number of neighbors for the minimum free energy structure [resp. the structure having minimum free energy among those structures that are consistent with the Rfam consensus structure]. EXP stands for the length-normalized expected number of neighbors, as computed by RNAexpNumNbors, and BPdist is the length-normalized base pair distance between the MFE structure and the CMFE structure. The table shows perhaps surprisingly that the MFE structure does not have significantly more neighbors than the Boltzmann expected number; however, the CMFE structure does. The Rfam consensus structure is often taken as the gold standard in RNA benchmarking studies; however, since Rfam base pairs are inferred only by covariation found in a multiple alignment, we take the CMFE structure as representative of the native structure. It appears significant that the CMFE structure has significantly more neighbors than the MFE structure. Another striking observation is large value of EXPB-MFE for the two riboswitch families present in the collection of Rfam sequences we investigated - purine riboswitch (RF00167) and molybdenum cofactor riboswitch (RF01055). If this finding holds up under careful scrutiny of all riboswitch families in Rfam, then perhaps RNAexpNumNbors could be used as a tool, along with RNAbor [7] and FFTbor [18] to detect conformational switches.

\section{Z-scores}

The left panel of Figure 5 depicts the relative frequency for the EXPB value for 1000 random RNAs, where EXPB denotes the length-normalized Boltzmann expected number of neighbors. The arrow head in the graph marks the EXPB value for wild type purine riboswitch with EMBL accession code AE005176.1/11595091159606. Random RNA sequences were generated to have the same dinucleotides as that of the wild type purine riboswitch by using the Altschul-Erikson algorithm [1. Wild type purine riboswitch with EMBL accession code AE005176.1/1159509-1159606 has EXPB value 0.782112, while the average EXPB of the 1000 randomized RNAs is 0.837026 with standard deviation 0.253572 . It follows that the Z-score for this riboswitch is $z=\frac{0.782112-0.837026}{0.253572}=-0.21656$ - i.e. most random RNAs have larger EXPB values than this riboswitch. This situation is in fact typical, as shown by the center panel of Figure 5, which depicts the relative frequency of Z-scores for the length-normalized expected number of neighbors for 133 purine riboswitch sequences from the seed alignment of Rfam family RF00167. For each riboswitch sequence, the expected number $x$ of neighbors was computed, and well as the expeccted number $x_{1}, \ldots, x_{100}$ of 100 random RNA sequences having the same dinucleotides, obtained by the Altschul-Erikson algorithm. Z-scores were computed as $z=\frac{x-\mu}{\sigma}$, where $\mu$ is the mean $\mu$ and $\sigma$ is the standard deviation $x_{1}, \ldots, x_{100}$. Z-scores computed with respect to the Boltzmann probability with overall mean -0.291 appear in blue, while those computed with respect to the uniform probability with overall mean +0.019 appear in red. It follows that purine riboswitches tend to have a lower expected number of neighbors than do their randomizations.

The right panel of Figure 5 depicts the relation between MFE-EXPB and its Z-scores, described as follows. For each purine riboswitch a, the length-normalized difference MFE-EXPB between the number of neighbors of the MFE structure and the Boltzmann expected number was computed, as well that for 100 random RNA sequences $\mathbf{a}_{1}, \ldots, \mathbf{a}_{100}$ having the same dinucleotides, obtained by the Altschul-Erikson algorithm. Let $x$ be the MFE-EXPB value for a), and let $\mu$ [resp. $\sigma$ ] denote the mean [resp. standard deviation] for the MFE-EXPB values of the random RNAs. The Z-scores $z=\frac{x-\mu}{\sigma}$ of purine riboswitches $\alpha$ are highly correlated with the values MFE-EXPB, with $r=0.9606$. The right panel of Figure 5 clearly indicates that MFE-EXPB for purine riboswitches is about two times larger in absolute value than than for randomized RNAs.

\section{Discussion}

Understanding the network topology of macromolecules is important, both for folding kinetics and for computational approaches to determine the native structure of protein and RNA. In this paper, we consider the 


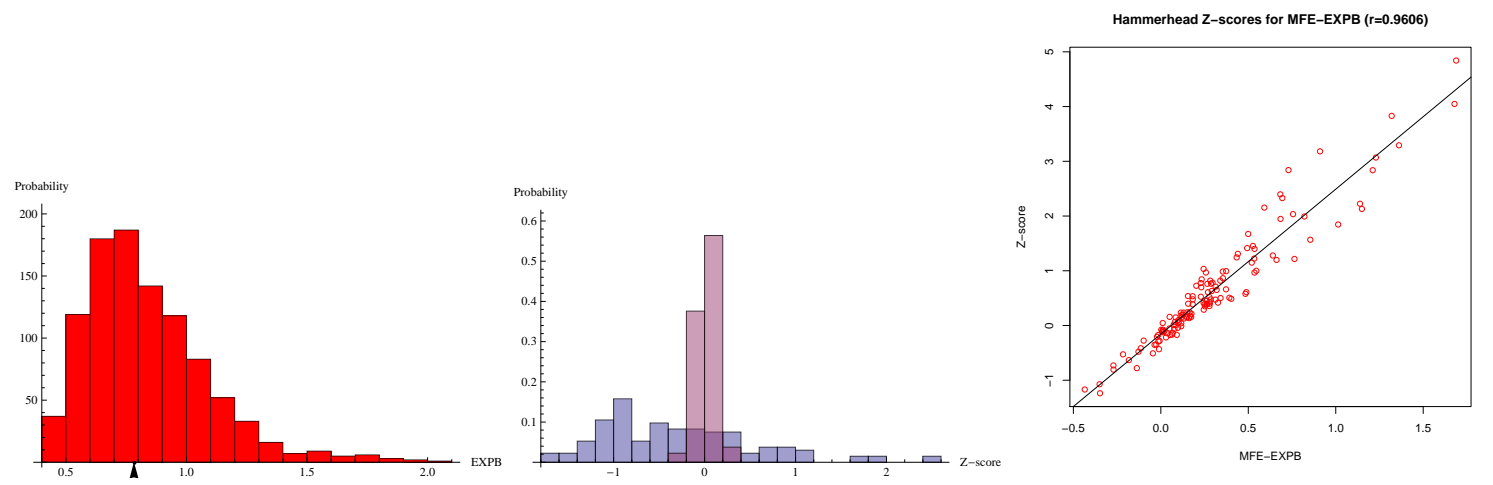

Figure 5: (Left) Distribution of length-normalized Boltzmann expected number of neighbors (EXPB) values for 1000 random RNAs, where the arrow head marks the EXPB value wild type purine riboswitch with EMBL accession code AE005176.1/1159509-1159606. Random RNA sequences were generated to have the same dinucleotides as that of the wild type purine riboswitch by using the Altschul-Erikson algorithm [1]. (Center) Histogram of Z-scores, given by the formula ('wild type EXPB' - 'mean EXPB of random RNA') divided by 'stdev EXPB of random RNA', for the length-normalized expected number of neighbors for the 133 purine riboswitches from the seed alignment of Rfam family RF00167. For each riboswitch, its expected number $x$ of neighbors was computed, as well as the mean $\mu$ and standard deviation $\sigma$ of the expected number of neighbors for 100 random RNA sequences having the same dinucleotides, obtained by the Altschul-Erikson algorithm. Z-scores for expectations with respect to the Boltzmann [resp. uniform] probability appear in blue [resp. red], with an overall mean of -0.291 [resp. +0.019]. (Right) Scatter plot of Z-scores for MFE-EXPB, where for a given RNA sequence a, MFE-EXPB is defined as the length-normalized number of neighbors of the MFE structure minus the length-normalized expected number of neighbors. For each riboswitch, MFE-EXPB was computed, as well as that for 100 random RNA sequences having the same dinucleotides, obtained by the Altschul-Erikson algorithm. Corresponding Z-scores $z=\frac{x-\mu}{\sigma}$ were computed, where $x$ is MFE-EXPB and $\mu, \sigma$ are respectively the mean, standard deviation of corresponding MFE-EXPB values for the randomized RNAs. Pearson correlation is 0.9606 . 
network of all secondary structures of a given RNA sequence, where nodes are connected when the corresponding structures have base pair distance one. We describe the first algorithm to compute the expected network degree, where expectation is computed with respect to either the uniform or Boltzmann probability. Using dynamic programming, our C-program RNAexpNumNbors runs in cubic time with quadratic memory requirements, although the network size is exponential in input RNA sequence length.

Our network is related, but different from that of [27, in which nodes (secondary structures) $s, t$ are linked by an edge if $t$ can be obtained from $s$ by adding or removing a base pair, or by by a shift move. In contrast to Wuchty's observation, that the clustering coefficient $C(\nu)$ for phe-tRNA is inversely proportional to node degree $k_{\nu}$ of node $\nu$, for our network, $C(\nu)=0$ for all nodes $\nu$. Indeed, if $s, t$ and $s, u$ are neighbors, then $d_{\mathrm{BP}}(s, t)=1$ and $d_{\mathrm{BP}}(s, u)=1$, so it follows that $d_{\mathrm{BP}}(t, u)=2$ - no two neighbors of $s$ are connected by an edge. At present, it is unclear whether RNAexpNumNbors can be extended to allow shift moves, a topic we hope to explore in future work.

Using RNAexpNumNbors, we analyzed a number of noncoding RNAs from the Rfam database. We have shown that for such RNAs, the expected degree is generally less than the degree of the minimum free energy (MFE) structure, which in turn is less than the degree of the minimum free energy structure constrained to be consistent with the Rfam consensus structure (CMFE). This observation is consistent with what one knows from Markov state models formed by clustering structures from molecular dynamics snapshots [2]. However, the expected degree of structural RNAs, such as purine riboswitches, paradoxically appears to be smaller than that of random RNA, yet the difference between the degree of the MFE structure and the expected degree is larger than that of random RNA. Expected degree does not seem to correlate with any structural diversity measure of RNA, such as positional entropy, ensemble defect, etc. Moreover, there is no correlation (-0.054286) between the expected number of neighbors and folding time, nor any corrlation (-0.036524) between the MFE-EXPB and folding time, as measured by Kinfold [6] using a carefully chosen benchmarking set of 1000 20-mers from [17].

We close this paper by posing a few questions. One can argue that the collection of neighbors of a given structure $s$ constitutes a conformational breathing space for thermal movement while retaining functionality. Hence, the term $S=k_{B} \cdot \log N(s)$ is a form of configurational entropy, which is not currently accounted for in RNA secondary structure models. Can one define the free energy term $c \cdot T \cdot S$, for absolute temperature $T$, where $c$ is an appropriate weight with respect to the Turner energy parameters? Would such an additional energy contribution improve structure prediction? Does expected network degree play a role in RNA molecular evolution? Can the algorithm RNAexpNumNbors be extended to allow shift moves, or to apply to $k$-neighbors, for $k>1$ ? Finally, in reference to Figure 4 can one prove the existence of an asymptotic limit $\lim _{|\mathbf{a}| \rightarrow \infty} \frac{\langle N(\mathbf{a})\rangle}{|\mathbf{a}|}$ for homopolymers a, using the algebraic combinatorial techniques of [5, 3, 8] ?

\section{Methods}

In this section, we provide recursions for efficient dynamic programming algorithms for the expected number $\left\langle N_{s}\right\rangle$ of neighbors of secondary structure $s$, where $s$ varies over all secondary structures of a given RNA sequence a. For clarity of exposition, we present three different algorithms, depending on the probability model for secondary structures (uniform model versus Boltzmann with Nussinov energy model versus Boltzmann with Turner energy model).

\subsection{Model A: uniform probability distribution}

In this subsection, $\left\langle N_{s}\right\rangle$ is formally defined as follows

$$
\left\langle N_{s}\right\rangle=\frac{\sum_{s} N_{s}}{Z}
$$

where $N_{s}$ denotes the number of secondary structures, whose base pair distance with $s$ is 1 , and $Z$ denotes the total number of secondary structures of given RNA sequence $\mathbf{a}$, and the summation is taken over all secondary structures $s$ of $\mathbf{a}$. For any secondary structure $s$, let $|s|$ denote the number of base pairs in $s$.

Suppose that $\mathbf{a}=\mathbf{a}_{1}, \ldots, \mathbf{a}_{n}$. For $1 \leq i \leq j \leq n$, define $\mathbf{a}[i, j]=\mathbf{a}_{i}, \ldots, \mathbf{a}_{j}$, and define $S S(\mathbf{a}[i, j])$ to be 
the collection of secondary structures of $\mathbf{a}[i, j]$. Define

$$
Q_{i, j}=\sum_{s \in S S(\mathbf{a}[i, j])} N_{s}
$$

Similarly, let $Z_{i, j}=\sum_{s \in S S(\mathbf{a}[i, j])} 1$; i.e. $Z_{i, j}$ denotes the number of secondary structures of $\mathbf{a}[i, j]$.

BASE CASE: For $j-i \in\{0,1,2,3\}, Q_{i, j}=0$ and $Z_{i, j}=1$.

Inductive CASE: Let $B P(i, j, \mathbf{a})$ be a boolean function, taking the value 1 if positions $i, j$ can form a base pair for sequence $\mathbf{a}$, and otherwise taking the value 0 . Assume that $j-i>3$.

Subcase A: Consider all secondary structures $s \in \mathbf{a}[i, j]$, for which $j$ is unpaired. For each structure $s$ in this subcase, the number $N_{s}$ of neighbors of $s$ is constituted from the number of structures obtained from $s$ by removal of a single base pair, together with the number of structures obtained from $s$ by addition of a single base pair. If the base pair added does not involve terminal position $j$, then total contribution to $\sum_{s \in S S(\mathbf{a}[i, j])} N_{s}$ is $Q_{i, j-1}$. It remains to count the contribution due to neighbors $t$ of $s$, obtained from $s \in S S(\mathbf{a}[i, j])$ by adding the base pair $(k, j)$. This contribution is given by $\sum_{k=i}^{j-4} B P(k, j, \mathbf{a}) \cdot Z_{i, k-1} \cdot Z_{k+1, j-1}$, where $Z_{i, i-1}$ is defined to be 1 . Thus the total contribution to $Q_{i, j}$ from this subcase is

$$
Q_{i, j-1}+\sum_{k=i}^{j-4} B P(k, j, \mathbf{a}) \cdot Z_{i, k-1} \cdot Z_{k+1, j-1} .
$$

Subcase B: Consider all secondary structures $s \in \mathbf{a}[i, j]$ that contain the base pair $(k, j)$ for some $k \in$ $\{i, \ldots, j-4\}$. For secondary structure $s$ in this subcase, the number $N_{s}$ of neighbors of $s$ is constituted from the number of structures obtained by removing base pair $(k, j)$ together with a contribution obtained by adding/removing a single base pair either to the region $[i, k-1]$ or to the region $[k+1, j-1]$. Setting $Q_{i, i-1}$ to be 0 , these contributions are given by

$$
\sum_{k=i}^{j-4} B P(k, j, \mathbf{a}) \cdot\left[Z_{i, k-1} \cdot Z_{k+1, j-1}+Q_{i, k-1} \cdot Z_{k+1, j-1}+Z_{i, k-1} \cdot Q_{k+1, j-1}\right] .
$$

In the current subcase, the contribution to $Z_{i, j}$ is $\sum_{k=i}^{j-4} B P(k, j, \mathbf{a}) \cdot Z_{i, k-1} \cdot Z_{k+1, j-1}$.

Finally, taking the contributions from both subcases together, it follows that

$$
\begin{aligned}
Q_{i, j} & =Q_{i, j-1}+\sum_{k=i}^{j-4} B P(k, j, \mathbf{a}) \cdot\left[2 \cdot Z_{i, k-1} \cdot Z_{k+1, j-1}+Q_{i, k-1} \cdot Z_{k+1, j-1}+Z_{i, k-1} \cdot Q_{k+1, j-1}\right] \\
Z_{i, j} & =Z_{i, j-1}+\sum_{k=i}^{j-4} B P(k, j, \mathbf{a}) \cdot Z_{i, k-1} \cdot Z_{k+1, j-1} .
\end{aligned}
$$

It follows that the expected number $\left\langle N_{s}\right\rangle$ of neighbors $N_{s}$ of structures $s$ of $\mathbf{a}$ is $\frac{Q_{1, n}}{Z_{1, n}}$.

We should remark that the recursion for $Z_{i, j}$ is well-known and due originally to Waterman, where in 21 the asymptotic number of secondary structures of a homopolymer is determined. However, to the best of our knowledge, the recursions and related dynamic programming algorithm for $\left\langle N_{s}\right\rangle$ are new. We have implemented the dynamic programming algorithm corresponding to equations (3) and (4), as well as an algorithm proceeding by brute force enumeration as a cross-check of the first algorithm. Subsequently, we have cross-checked the recursions for Models B and C by setting energy terms to zero and comparing the results with our implementation for Model A.

Now we give the recursions for a dynamic programming algorithm to compute $\langle N\rangle=\sum_{s} P(s) \cdot N(s)$, where the sum is taken over all secondary structures $s$ of the input RNA sequence $\mathbf{a}=a_{1}, \ldots, a_{n}, N(s)$ is the number of structures of a that differ by one base pair from $s$, and $P(s)=\sum_{\exp (-E(s) / R T)}$ is the Boltzmann probability of structure $s$, where $E(s)$ is alternately the Nussinov base pairing energy model or the Turner base stacking energy model. We provide derivations for the recursions in the Appendix. 


\section{Model B: Base pairing energy}

Here we consider the Nussinov energy model [15], where each base pair of a secondary structure contributes an energy of -1 . It follows that for secondary structure $s$ of $\mathbf{a}=\mathbf{a}_{1}, \ldots, \mathbf{a}_{n}, E(s)=-1 \cdot|s|$, where $|s|$ denotes the number of base pairs in $s$. For this model, the expected number of neighbors $\left\langle N_{s}\right\rangle$ is defined by

$$
\left\langle N_{s}\right\rangle=\frac{\sum_{s \in S S(\mathbf{a})} N_{s} \cdot \exp (-E(s) / R T)}{Z}=\sum_{s \in S S(\mathbf{a})} N_{s} \cdot P(s)
$$

where $P(s)=\frac{\exp (-E(s) / R T)}{Z}$ denotes the Boltzmann probability of structure $s$. (In the previous section, the uniform probability of $s$ was $1 / Z$, where $Z$ denoted the number of structures.) In contrast to the previous subsection, here we define $Q, Z$ as follows

$$
\begin{aligned}
Q_{i, j} & =\sum_{s \in S S(\mathbf{a}[i, j])} N_{s} \cdot \exp (-E(s) / R T) \\
Z_{i, j} & =\sum_{s \in S S(\mathbf{a}[i, j])} \exp (-E(s) / R T)
\end{aligned}
$$

In [15, the energy function $E_{i, j}$ is defined to be -1 if positions $i, j$ can form a base pair, and otherwise $E_{i, j}=0$. A slightly better refinement is the following energy function that one could adopt:

$$
E_{i, j}= \begin{cases}-3 & \text { if } a_{i}, a_{j}=G C \text { or } a_{i}, a_{j}=C G \\ -2 & \text { if } a_{i}, a_{j}=A U \text { or } a_{i}, a_{j}=U A \\ -1 & \text { if } a_{i}, a_{j}=G U \text { or } a_{i}, a_{j}=U G \\ 0 & \text { otherwise. }\end{cases}
$$

BASE CASE: For $1 \leq i \leq n$ and $j=i-1$, define $Z_{i, j}=1$ and $Q_{i, j}=0$. For $j \in\{i, \ldots, i+3\}$, define $Z_{i, j}=1$ and $Z B_{i, j}=Z M_{i, j}=Z M 1_{i, j}=0$ and $Q B_{i, j}=Q M_{i, j}=Q M 1_{i, j}=0$.

InduCtive CASE: For $i+3<j$,

$$
\begin{aligned}
Q_{i, j}= & Q_{i, j-1}+\sum_{k=i}^{j-4} b p(k, j) \cdot\left[Z_{i, k-1} Z_{k+1, j-1}\left(1+\exp \left(\frac{-E_{k, j}}{R T}\right)\right)\right]+ \\
& \sum_{k=i}^{j-4} b p(k, j) \cdot \exp \left(\frac{-E_{k, j}}{R T}\right) \cdot\left[Q_{i, k-1} Z_{k+1, j-1}+Z_{i, k-1} Q_{k+1, j-1}\right] \\
Z_{i, j}= & Z_{i, j-1}+\sum_{k=i}^{j-4} b p(k, j) \cdot \exp \left(\frac{-E_{k, j}}{R T}\right) \cdot Z_{i, k-1} \cdot Z_{k+1, j-1} .
\end{aligned}
$$

\section{Model C: Turner energy}

Define the following helper functions:

$$
\begin{aligned}
\operatorname{arc} 1(i, j) & =|\{(x, y): b p(x, y)=1, i \leq x<y \leq j, x+3<y\}| \\
\operatorname{arc} 2(i, j, \ell, r) & =|\{(x, y): b p(x, y)=1, i<x<\ell, r<y<j\}| \\
\operatorname{arc} 3(i, j, \ell, r) & =\operatorname{arc1}(i+1, \ell-1)+\operatorname{arc1}(r+1, j-1)+\operatorname{arc2}(i, j, \ell, r) .
\end{aligned}
$$

Note the occurrence of inequality $\leq$ in $\operatorname{arc} 1$, in contrast to the occurrence of strict inequality $<$ in $\operatorname{arc} 2$. Clearly, $\operatorname{arc} 1(i, j)$ is the number of potential base pairs in the input RNA sequence $a_{1}, \ldots, a_{n}$ that are found in the interval $[i, j]$. In contrast, $\operatorname{arc} 2(i, j, \ell, r)$ is the number of potential base pairs $(x, y)$, where $x$ occurs in the left bulge and $y$ occurs in the right bulge of a reference structure; i.e. the number of potential base pairs that 'bridge' an internal loop. Finally, $\operatorname{arc} 3(i, j, \ell, r)$ is the number of potential base pairs occurring in the 
left bulge, right bulge or 'bridging' the internal loop. Of course, it is possible that $\ell=i+1$ [resp. $r=j-1$ ], in which case there is no left bulge [resp. right bulge] and hence no internal loop.

BASE CASE: For $1 \leq i \leq n$ and $j=i-1$, define $Z_{i, j}=1$ and $Q_{i, j}=0$. For $j \in\{i, \ldots, i+3\}$, define $Z_{i, j}=1$ and $Z B_{i, j}=Z M_{i, j}=Z M 1_{i, j}=0$ and $Q B_{i, j}=Q M_{i, j}=Q M 1_{i, j}=0$.

InduCtive CASE: For $i+3<j$,

$$
\begin{aligned}
Q_{i, j} & =Q_{i, j-1}+\sum_{k=i}^{j-4} b p(k, j) \cdot\left[Z_{i, k-1} Z_{k+1, j-1}+Q_{i, k-1} Z B_{k, j}+Z_{i, k-1} Q B_{k, j}\right] \\
Q B_{i, j}= & A_{i, j}+B_{i, j}+C_{i, j} \\
A_{i, j}= & \exp \left(-\frac{H(i, j)}{R T}\right) \cdot[1+\operatorname{arc} 1(i+1, j-1)] \\
B_{i, j}= & \sum_{\ell=i+1}^{\min (i+31, j-5) \max (j-31, i+5)} \exp \left(-\frac{I(i, j, \ell, r)}{R T}\right) \cdot\left[Z B_{\ell, r}(1+\operatorname{arc} 3(i, j, \ell, r))+Q B_{\ell, r}\right] \\
C_{i, j}= & \sum_{r=i+5}^{j-5} \exp \left(-\frac{a+b}{R T}\right) \cdot\left[Q M_{i+1, r-1} Z M 1_{r, j-1}+Z M_{i+1, r-1} Q M 1_{r, j-1}\right] \\
Q M 1_{i, j}= & \sum_{k=i+4}^{j} \exp \left(-\frac{c(j-k)}{R T}\right) \cdot\left[Q B_{i, k}+Z B_{i, k} \cdot \operatorname{arc} 1(k+1, j)\right] \\
Q M_{i, j}= & \sum_{r=i}^{j-5} \exp \left(-\frac{b+c(r-i)}{R T}\right) \cdot Q M 1_{r, j}+Z M 1_{r, j} \cdot \operatorname{arc} 1(i, r-1)+ \\
& \sum_{r=i}^{j-5}\left[Q M_{i, r-1} Z M 1_{r, j}+Z M_{i, r-1} Q M 1_{r, j}\right]
\end{aligned}
$$

Finally, to accelerate the computation of the functions $\operatorname{arc} 1, \operatorname{arc} 2$, the $4 \times n \times n$ array $A R C$ should be precomputed, where if $\mathbf{a}=a_{1}, \ldots, a_{n}$ denotes the input RNA sequence, then

$$
A R C[\alpha, i, j]= \begin{cases}\left|x \in[i, j]: a_{x}=U\right| & \text { if } \alpha=0 \\ \left|x \in[i, j]: a_{x}=G\right| & \text { if } \alpha=1 \\ \left|x \in[i, j]: a_{x} \in\{C, U\}\right| & \text { if } \alpha=2 \\ \left|x \in[i, j]: a_{x} \in\{A, G\}\right| & \text { if } \alpha=3 .\end{cases}
$$

If $\operatorname{index}(\alpha)=0,1,2,3$ respectively for values $\alpha=A, C, G, U$, then $\operatorname{arc} 1(i, j)=\sum_{k=i}^{j-4} A R C\left[\operatorname{index}\left(a_{k}\right), k+4, j\right]$ and $\operatorname{arc} 2(i, j, \ell, r)=\sum_{k=i+1}^{\ell-1} A R C\left[\operatorname{index}\left(a_{k}\right), r+1, j-1\right]$.

Note that in the implementation of $B_{i, j}$, the first sum $\sum_{\ell=i+1}^{\min (i+31, j-5)}$ is implemented by the FOR loop

for $\ell=i+1$ to $\min (i+31, j-5)$

while the second sum $\sum_{r=j-1}^{\max (j-31, i+5)}$ is implemented by the reverse FOR loop

for $r=j-1$ down to $\max (j-31, i+5)$

and although not written explicitly in the expression for $B_{i, j}$, there is a check that $(\ell-i-1)+(j-r-1) \leq 30$. This follows the convention in Vienna RNA Package that internal loops have size bounded by 30.

It is worth noting that if all energy terms are set to zero, then $Q_{i, j}$ in this section is not necessarily equal to $N_{i, j}$, in the treatment of the uniform probability case. This is because we have ignored structural neighbors formed by addition of a base pair $(x, y)$ in a multiloop structure $s$ closed by base pair $(i, j)$, where $i<x<\ell<r<y<j$, while $(\ell, r) \in s$; i.e. the base pair $(x, y)$ spans one or more components of a multiloop and connects the previously unpaired positions $x, y$ in the multiloop $s$. We are obliged to ignore such potential structural neighbors because of technical treatment of multiloops in the McCaskill partition function [14. Nevertheless, when using the Turner energy parameters, in practice there should be only a 
small discrepancy with the true value of $Q_{i, j}$ as computed by brute force. This is because we expect both the number of unpaired bases and the number of components in a multiloop to be small, so there will be few occasions where this special case might arise (otherwise, this is energetically unfavorable, and hence the Boltzmann probability would be small).

\section{Acknowledgements}

The work reported in this paper was done during a visit with Niles Pierce at the California Institute of Technology, Knut Reinert at the Free University of Berlin and Martin Vingron at the Max Planck Institute for Molecular Genetics. Warm thanks are due to all three persons. We would like to thank the reviewers for their very helpful comments. This research was funded by a Guggenheim Fellowship, National Science Foundation grant DBI-1262439, and funding from the Deutscher Akademischer Austauschdienst. Any opinions, findings, and conclusions or recommendations expressed in this material are those of the authors and do not necessarily reflect the views of the National Science Foundation. 


\section{Appendix}

\section{RNA structural measures}

In the main text, we determined the correlation between the expected network degree and the following RNA structural measures: expected number of base pairs, expected base pair distance, ensemble defect, positional entropy. In our correlations in the main text, each of these measures was length-normalized by dividing the value by sequence length. These structural measures can be defined from the base pairing probabilities, computed by McCaskill's algorithm [14] and implemented in RNAfold -p [1]. Let

$$
p_{i, j}=\sum_{\{s:(i, j) \in s\}} P(s)=\frac{\sum_{\{s:(i, j) \in s\}} \exp (-E(s) / R T)}{Z}
$$

where $P(s)$ is the Boltzmann probability of structure $s$ of a given RNA sequence $\mathbf{a}=\mathbf{a}_{1}, \ldots, \mathbf{a}_{n}, E(s)$ is the Turner 1999 energy of secondary structure $s\left[13,29, R \approx 0.001987 \mathrm{kcal} \mathrm{mol}^{-1} \mathrm{~K}^{-1}\right.$ is the universal gas constant, $T=310.15$ is absolute temperature, and the partition function $Z=\sum_{s} \exp (-E(s) / R T)$, where the sum is taken over all secondary structures $s$ of $\mathbf{a}$. Symmetrize the base pair probabilities, by defining $p_{j, i}=p_{i, j}$ for $1 \leq i, j \leq n, i \neq j$, and define $p_{i, i}=1-\sum_{i \neq j} p_{i, j}$ to be the probability that position $i$ is unpaired. Let $s_{0}$ denote the minimum free energy structure of input RNA sequence $\mathbf{a}$.

1. Expected number of base pairs (ExpNumBP) is defined by $\sum_{1 \leq i<j \leq n} p_{i, j}$.

2. Expected base pair distance (ExpBPDist) to the MFE structure $s_{0}$ of input RNA sequence a is defined by $\sum_{1 \leq i<j \leq n} I\left[(i, j) \notin s_{0}\right] \cdot p_{i, j}+I\left[(i, j) \in s_{0}\right] \cdot\left(1-p_{i, j}\right)$, where $I$ denotes the indicator function.

3. Ensemble defect (EnsDef) is the expected number of nucleotides whose base pairing status differs from the MFE structure $s_{0}$, defined by $n-\sum_{i \neq j} p_{i, j} \cdot I\left[(i, j) \in s_{0}\right]-\sum_{1 \leq i \leq n} p_{i, i} \cdot I\left[i\right.$ unpaired in $\left.s_{0}\right]$, where $I$ is the indicator function.

4. Total positional entropy $(\mathrm{H})$ is defined by $\langle H(\mathbf{a})\rangle=\sum_{i=1}^{n}\left\{-\left(p_{i, i} \cdot \ln p_{i, i}+\left(1-p_{i, i}\right) \cdot \ln \left(1-p_{i, i}\right)\right)\right\}$, where $0 \cdot \ln 0$ is defined to be 0 .

\section{Expected number of neighbors for Boltzmann distribution}

In this section, we provide full details on the derivation of the recursions from Section 5 . By setting all energy terms to zero in the recursions for Models A, B and C, we should obtain the same value as in the uniform probability case. In testing RNAexpNumNbors, this is indeed the case, except for a very slight undercount in multiloops in Model C. For the reasons explained at the end of the Appendix, this will make little difference when using the Turner energy parameters, since multiloops are energetically unfavorable.

Throughout this section, $\mathbf{a}=a_{1}, \cdots, a_{n}$ denotes an arbitrary but fixed RNA sequence. Below, we justify the recursions given for $\langle Q(s)\rangle=\sum_{s} B F(s) \cdot N(s)$, where the sum is taken over all secondary structures $s$ of RNA sequence $\mathbf{a}, N(s)$ is the number of structures of a that differ by one base pair from $s$, and the Boltzmann factor $B F(s)$ of $s$ is defined by $\exp (-E(s) / R T)$, where $E(s)$ is the free energy of $s$. Recursions are also given for the partition function $Z(s)=\sum_{s} \exp (-E(s) / R T)$, where the sum is taken over all secondary structures of $\mathbf{a}$. It follows that the expected number of structural neighbors

$$
\langle N\rangle=\sum_{s} B F(s) \cdot N(s)=\frac{Q(s)}{Z(s)}
$$

For $1 \leq i \leq j \leq n$, the collection of all secondary structures of $\mathbf{a}[i, j]=a_{i}, \ldots, a_{j}$ is denoted $s s[i, j]$. In contrast, if $s$ is a secondary structure of $a_{1}, \ldots, a_{n}$, then $s[i, j]$ is the restriction of $s$ to the interval $[i, j]$, defined by $s[i, j]=\{(x, y): i \leq x \leq y \leq j,(x, y) \in s$.

We give separate algorithms for the expected number $\langle N\rangle$ of structural neighbors, depending on whether the free energy $E(s)$ is computed with respect to the Nussinov base pairing energy model or the Turner base 
stacking energy model. However, the initial portion of the derivation is common to both energy models. Define

$$
\begin{aligned}
Q B_{i, j} & =\sum_{\substack{s \in s s[i, j] \\
(i, j) \in s}} B F(s) N(s) \\
Z B_{i, j} & =\sum_{\substack{s \in s s[i, j] \\
(i, j) \in s}} B F(s) .
\end{aligned}
$$

\subsection{Initial derivation shared by energy Model B and C}

For notational convenience, we define $Q_{i, i-1}=0$ and $Z_{i, i-1}=1$. If $i \leq j<i+4$, then for any secondary structure $s$, there are no structural neighbors of $s[i, j]$ and so $Q_{i, j}=0$. As well, the only secondary structure on $[i, j]$ is the empty structure, so $Z_{i, j}=1$.

Now assume that $i+4 \leq j$. Since

$$
Q_{i, j}=\sum_{\substack{s \in s s[i, j] \\ \text { unpaired in } s}} B F(s) N(s)+\sum_{k=i}^{j-4} \sum_{\substack{s \in s s[i, j] \\(k, j) \in s}} B F(s) N(s) .
$$

we treat each sum in a separate case. Let $b p(k, j)$ be a boolean valued function with the value 1 if $k$ can base-pair with $j$; i.e. $a_{k} a_{j} \in\{A U, U A, C G, G C, G U, U G\}$. For secondary structure $s \in s s[i, j]$, let $b p(k, j, s)$ be a boolean function with value 1 if it is possible to add the base pair $(k, j)$ to $s$ and obtain a valid secondary structure; i.e. without creating a base triple or pseudoknot.

CASE 1: $j$ is unpaired in $[i, j]$. For $s \in s s[i, j]$ in which $j$ is unpaired, $s=s[i, j-1]$ and $B F(s)=$ $B F(s[i, j-1])$. The contribution to $Q_{i, j}$ in this case is given by

$$
\begin{aligned}
A_{i, j} & =\sum_{\substack{s \in s s[i, j] \\
\text { unpaired in } s}} B F(s) N(s) \\
& =\sum_{s \in s s[i, j-1]} B F(s)\left[N(s)+\sum_{i \leq k \leq j-4, k \text { unpaired in } s} 1\right] \\
& =Q_{i, j-1}+\sum_{k=i}^{j-4} b p(k, j) \sum_{s_{1} \in s s[i, k-1]} \sum_{s_{2} \in s s[k+1, j-1]} \\
& =Q_{i, j-1}+\sum_{k=i}^{j-4} b p(k, j) \cdot Z_{i, k-1} \cdot Z_{k+1, j-1}
\end{aligned}
$$

The term 1 on the right side of line 2 arises from neighbors of $s$ obtained by adding the base pair $(k, j)$ to $s$. In line 3 , note that if $s \in s s[i, j]$ is a structure in which both $k, j$ are unpaired and $b p(k, j, s)=1$, then $B F(s)=B F\left(s_{1}\right) \cdot B F\left(s_{2}\right)$. 
CASE 2: $j$ is paired in $[i, j]$. The contribution to $Q_{i, j}$ in this case is given by

$$
\begin{aligned}
& B_{i, j}=\sum_{k=i}^{j-4} \sum_{\substack{s \in s s[i, j] \\
(k, j) \in s}} B F(s) N(s)=\sum_{k=i}^{j-4} \sum_{\substack{s \in s s[i, j] \\
(k, j) \in s}} B F(s)[N(s[i, k-1])+N(s[k, j])] \\
& =\sum_{k=i}^{j-4} b p(k, j) \cdot\left\{\sum_{s_{1} \in s s[i, k-1]} \sum_{\substack{s_{2} \in s s[k, j] \\
(k, j) \in s_{2}}} B F\left(s_{1}\right) \cdot B F\left(s_{2}\right)\left[N\left(s_{1}\right)+N\left(s_{2}\right)\right]\right\} \\
& =\sum_{k=i}^{j-4} b p(k, j) \cdot\left\{\sum_{s_{1} \in s s[i, k-1]} B F\left(s_{1}\right) N\left(s_{1}\right) \sum_{\substack{s_{2} \in s s[k, j] \\
(k, j) \in s_{2}}} B F\left(s_{2}\right)+\right. \\
& \left.\sum_{s_{1} \in s s[i, k-1]} B F\left(s_{1}\right) \sum_{\substack{s_{2} \in s s[k, j] \\
(k, j) \in s_{2}}} B F\left(s_{2}\right) N\left(s_{2}\right)\right\} \\
& =\sum_{k=i}^{j-4} b p(k, j) \cdot\left\{Q_{i, k-1} \cdot Z B_{k, j}+Z_{i, k-1} \cdot Q B_{k, j}\right\} .
\end{aligned}
$$

Putting together the contributions from both cases, we have

$$
Q_{i, j}=Q_{i, j-1}+\sum_{k=i}^{j-4} b p(k, j)\left[Z_{i, k-1} Z_{k+1, j-1}+Q_{i, k-1} Z B_{k, j}+Z_{i, k-1} Q B_{k, j}\right] .
$$

\subsection{Model B: Base pairing energy}

In the Nussinov energy model, where the energy of base pair $(k, j)$ is denoted $E(k, j)$, we clearly have the following.

$$
\begin{aligned}
Q B_{i, j} & =\sum_{\substack{s \in s s[i, j] \\
(i, j) \in s}} B F(s) N(s) \\
& =\sum_{\substack{s \in s s[i, j] \\
(i, j) \in s}} B F(s)[1+N(s[i+1, j-1])] \\
& =Z B_{i, j}+\exp (-E(i, j) / R T) \cdot Q_{i+1, j-1} \\
Z B_{i, j} & =\exp (-E(i, j) / R T) \cdot Z_{i+1, j-1} .
\end{aligned}
$$

The term 1 arises from those neighbors of $s$, obtained by removal of the base pair $(i, j)$ while the term $N(s[i+1, j-1])$ arises from neighbors of $s$ obtained by removal/addition of a base pair within $[i+1, j-1]$. 
We now substitute the expressions for $Q B_{k, j}$ and $Z B_{k, j}$ into equation 10 .

$$
\begin{aligned}
Q_{i, j}= & Q_{i, j-1}+\sum_{k=i}^{j-4} b p(k, j)\left[Z_{i, k-1} Z_{k+1, j-1}+Q_{i, k-1} Z B_{k, j}+Z_{i, k-1} Q B_{k, j}\right] \\
= & Q_{i, j-1}+\sum_{k=i}^{j-4} b p(k, j)\left\{Z_{i, k-1} Z_{k+1, j-1}+Q_{i, k-1} Z_{k+1, j-1} \cdot \exp \left(-\frac{E(k, j)}{R T}\right)+\right. \\
& \left.Z_{i, k-1}\left[Z_{k+1, j-1} \cdot \exp \left(-\frac{E(k, j)}{R T}\right)+Q_{k+1, j-1} \cdot \exp \left(-\frac{E(k, j)}{R T}\right)\right]\right\} \\
= & Q_{i, j-1}+\sum_{k=i}^{j-4} b p(k, j)\left\{Z_{i, k-1} Z_{k+1, j-1}\left(1+\exp \left(-\frac{E(k, j)}{R T}\right)\right)+\right. \\
& \left.\exp \left(-\frac{E(k, j)}{R T}\right) \cdot\left[Q_{i, k-1} Z_{k+1, j-1}+Z_{i, k-1} Q_{k+1, j-1}\right]\right\}
\end{aligned}
$$

Note that if we set all base pair energies $E(k, j)$ to 0 , then we obtain the same expression as derived for the uniform probability distribution.
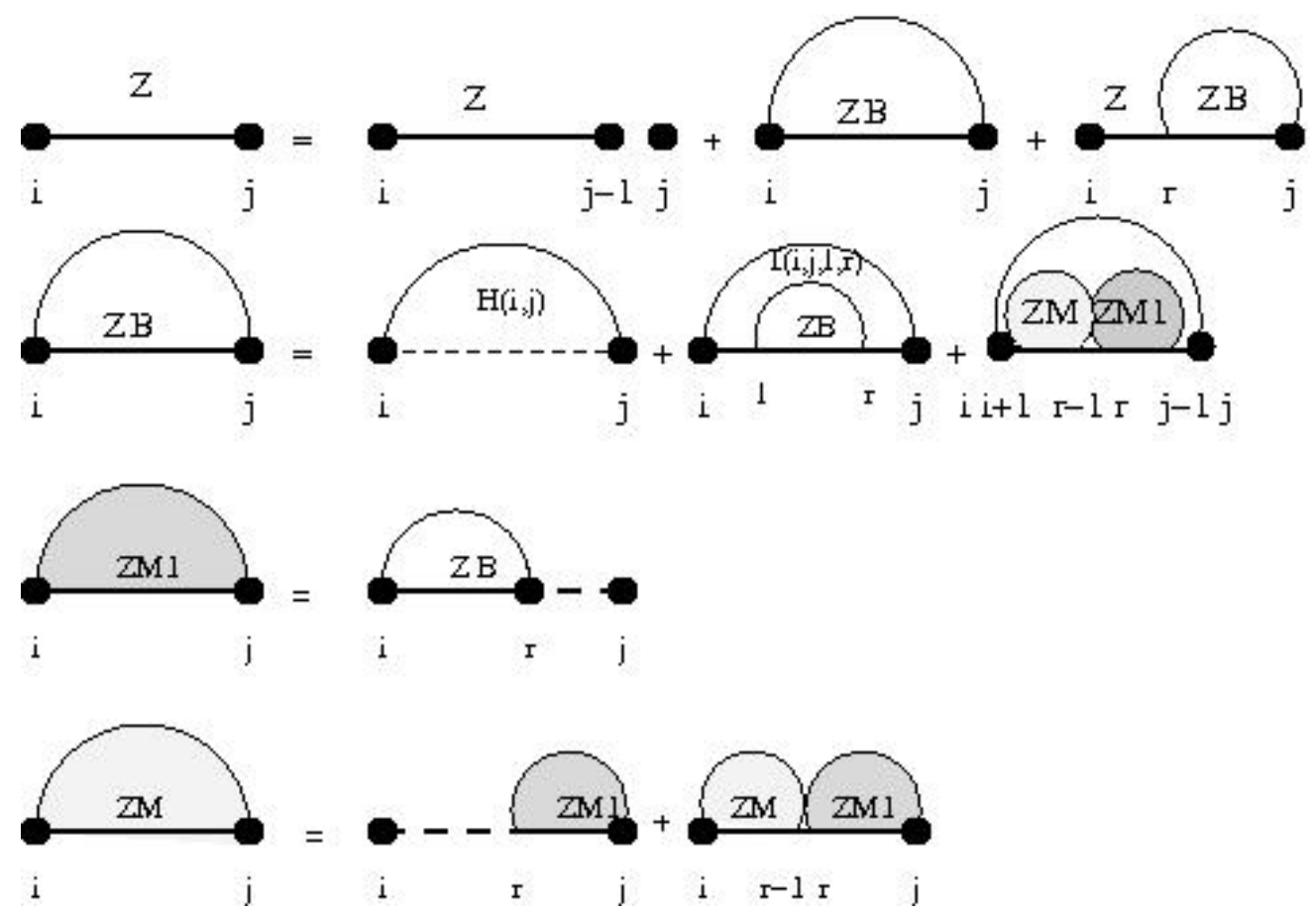

Figure 6: Feynman diagram which illustrates the recursions for McCaskill's algorithm [14].

\subsection{Model C: Turner nearest neighbor energy model}

In the nearest neighbor energy model [30, 22, free energies are defined not for base pairs, but rather for loops in the loop decomposition of a secondary structure. In particular, there are stabilizing, negative free energies for stacked base pairs and destabilizing, positive free energies for hairpins, bulges, internal loops, and multiloops.

In this section, free energy parameters for base stacking and loops are from the Turner 2004 energy model 22. As in the previous subsection, $Q, Z$ are defined, but now with respect to the Turner model, rather than 
the Nussinov model.

$$
\begin{aligned}
Q_{i, j} & =\sum_{s \in s s[i, j]} N_{s} \cdot \exp (-E(s) / R T) \\
Z_{i, j} & =\sum_{s \in s s[i, j]} \exp (-E(s) / R T)
\end{aligned}
$$

It follows that $Z=Z_{1, n}$ is the partition function for secondary structures (the Boltzmann weighted counting of all structures of $\mathbf{a}$ ) and

$$
\left\langle N_{s}\right\rangle=\frac{Q_{1, n}}{Z_{1, n}}=\sum_{s \in s s[1, n]} N_{s} \cdot P(s)=\sum_{s \in s s[1, n]} N_{s} \cdot \frac{\exp (-E(s) / R T)}{Z} .
$$

To complete the derivation of recursions for $\left\langle N_{s}\right\rangle$, we must define $Q B_{i, j}$ and $Z B_{i, j}$ in equation 100 for the Turner model.

To provide a self-contained treatment, we recall McCaskill's algorithm [14, which efficiently computes the partition function. For RNA nucleotide sequence $\mathbf{a}=\mathbf{a}_{1}, \ldots, \mathbf{a}_{n}$, let $H(i, j)$ denote the free energy of a hairpin closed by base pair $(i, j)$, while $I L\left(i, j, i^{\prime}, j^{\prime}\right)$ denotes the free energy of an internal loop enclosed by the base pairs $(i, j)$ and $\left(i^{\prime}, j^{\prime}\right)$, where $i<i^{\prime}<j^{\prime}<j$. Internal loops comprise the cases of stacked base pairs, left/right bulges and proper internal loops. The free energy for a multiloop containing $N_{b}$ base pairs and $N_{u}$ unpaired bases is given by the affine approximation $a+b N_{b}+c N_{u}$.

\section{Definition 1 (Partition function $Z$ and related function $Q$ )}

- $Z_{i, j}=\sum_{s} \exp (-E(s) / R T)$ where the sum is taken over all structures $s \in s s[i, j]$.

- $Z B_{i, j}=\sum_{s} \exp (-E(s) / R T)$ where the sum is taken over all structures $s \in s s[i, j]$ which contain the base pair $(i, j)$.

- $Z M_{i, j}=\sum_{s} \exp (-E(s) / R T)$ where the sum is taken over all structures $s \in s s[i, j]$ which are contained within an enclosing multiloop having at least one component.

- $Z M 1_{i, j}=\sum_{s} \exp (-E(s) / R T)$ where the sum is taken over all structures $s \in s s[i, j]$ which are contained within an enclosing multiloop having exactly one component. Moreover, it is required that $(i, r)$ is a base pair of $x$, for some $i<r \leq j$.

- $Q_{i, j}=\sum_{s} N_{s} \cdot \exp (-E(s) / R T)$ where the sum is taken over all structures $s \in s s[i, j]$.

- $Q B_{i, j}=\sum_{s} N_{s} \cdot \exp (-E(s) / R T)$ where the sum is taken over all structures $s \in s s[i, j]$ which contain the base pair $(i, j)$.

- $Q M_{i, j}=\sum_{s} N_{s} \cdot \exp (-E(s) / R T)$ where the sum is taken over all structures $s \in s s[i, j]$ which are contained within an enclosing multiloop having at least one component.

- $Q M 1_{i, j}=\sum_{s} N_{s} \cdot \exp (-E(s) / R T)$ where the sum is taken over all structures $s \in s s[i, j]$ which are contained within an enclosing multiloop having exactly one component. Moreover, it is required that $(i, r)$ is a base pair of $s$, for some $i<r \leq j$.

For $j-i \in\{0,1,2,3\}, Z(i, j)=1$, since the empty structure is the only possible secondary structure. for 
$j-i>3$, we have

$$
\begin{aligned}
Z_{i, j}= & Z_{i, j-1}+Z B_{i, j}+\sum_{r=i+1}^{j-4} Z_{i, r-1} \cdot Z B_{r, j} \\
Z B_{i, j}= & \exp (-H P(i, j) / R T)+\sum_{i \leq \ell \leq r \leq j} \exp (-I L(i, j \ell, r) / R T) \cdot Z B_{\ell, r}+ \\
& \exp (-(a+b) / R T) \cdot\left(\sum_{r=i+1}^{j-\theta-2} Z M_{i+1, r-1} \cdot Z M 1_{r, j-1}\right) \\
Z M 1_{i, j}= & \sum_{r=i+\theta+1}^{j} Z B_{i, r} \cdot \exp (-c(j-r) / R T) \\
Z M_{i, j}= & \sum_{r=i}^{j-\theta-1} Z M 1_{(r, j} \cdot \exp (-(b+c(r-i)) / R T)+ \\
& \sum_{r=i+\theta+2}^{j-\theta-1} Z M_{i, r-1} \cdot Z M 1_{r, j} \cdot \exp (-b / R T)
\end{aligned}
$$

See Figure 6 for a pictorial representation of the recursions of McCaskill's algorithm [14,

BASE CASE: For $j-i \in\{-1,0,1,2,3\}, Q_{i, j}=Q B_{i, j}=0, Z_{i, j}=1, Z B_{i, j}=Z M_{i, j}=Z M 1_{i, j}=0$.

Inductive Case: Assume that $j-i>3$. Define

$$
\begin{aligned}
\operatorname{arc} 1(i, j) & =|\{(x, y): b p(x, y)=1, i \leq x<y \leq j, x+3<y\}| \\
\operatorname{arc} 2(i, j, \ell, r) & =|\{(x, y): b p(x, y)=1, i<x<\ell<r<y<j\}| \\
\operatorname{arc} 3(i, j, \ell, r) & =\operatorname{arc1}(i+1, \ell-1)+\operatorname{arc1}(r+1, j-1)+\operatorname{arc} 2(i, j, \ell, r) .
\end{aligned}
$$

CASE A: $(i, j)$ closes a hairpin.

In this case, the contribution to $Q B_{i, j}$ is given by

$$
A_{i, j}=\exp \left(-\frac{H(i, j)}{R T}\right) \cdot[1+\operatorname{arc} 1(i+1, j-1)] .
$$

The term 1 arises from the neighbor of $s=\{(i, j)\}$ by removing base pair $(i, j)$. The term $\operatorname{arc} 1(i, j)$ arises from neighbors of $s$ obtained by adding a base pair in the region $[i+1, j-1]$.

CASE B: $(i, j)$ closes a stacked base pair, bulge or internal loop, whose other closing base pair is $(\ell, r)$, where $i<\ell<r<j$.

In this case, the contribution to $Q B_{i, j}$ is given by the following

$$
\begin{aligned}
& B_{i, j}=\sum_{\ell=i+1}^{\min (i+31, j-5)} \sum_{r=j-1}^{\max (j-31, i+5)} \exp \left(-\frac{I L(i, j, \ell, r)}{R T}\right) \cdot \sum_{\substack{s \in s s[\ell, r] \\
(\ell, r) \in s}} B F(s)[1+\operatorname{arc} 3(i, j, \ell, r)+N(s)] \\
& =\sum_{\ell=i+1}^{\min (i+31, j-5)} \sum_{r=j-1}^{\max (j-31, i+5)} \exp \left(-\frac{I L(i, j, \ell, r)}{R T}\right) \cdot Z B_{\ell, r} \cdot[1+\operatorname{arc} 3(i, j, \ell, r)]+ \\
& \sum_{\ell=i+1}^{\min (i+31, j-5)} \sum_{r=j-1}^{\max (j-31, i+5)} \exp \left(-\frac{I L(i, j, \ell, r)}{R T}\right) \cdot Q B_{\ell, r} \\
& =\sum_{\ell=i+1}^{\min (i+31, j-5)} \sum_{r=j-1}^{\max (j-31, i+5)} \exp \left(-\frac{I L(i, j, \ell, r)}{R T}\right) \cdot\left[Z B_{\ell, r} \cdot(1+\operatorname{arc3}(i, j, \ell, r))+Q B_{\ell, r}\right] .
\end{aligned}
$$


In the summation notation $\sum_{i=a}^{b}$, if upper bound $b$ is smaller than lower bound $a$, then we intend a loop of the form: FOR $i=b$ downto $a$.

CASE C: $(i, j)$ closes a multiloop.

In this case, the contribution to $Q B_{i, j}$ is given by the following

$$
\begin{aligned}
& C_{i, j}=\sum_{\substack{s \in s s[i, j],(i, j) \in s \\
(i, j) \text { closes a multiloop }}} B F(s) N(s) \\
& =\sum_{r=i+5}^{j-5} \exp \left(-\frac{a+b}{R T}\right) \cdot \sum_{\substack{s_{1} \in s s[i+1, r-1], s_{2} \in s s[r, j-1] \\
r \text { base-paired in } s_{2}}} B F\left(s_{1}\right) \cdot B F\left(s_{2}\right) \cdot\left[1+N\left(s_{1}\right)+N\left(s_{2}\right)\right] \\
& =\sum_{r=i+5}^{j-5} \exp \left(-\frac{a+b}{R T}\right) \cdot \sum_{s_{1} \in s s[i+1, r-1]} B F\left(s_{1}\right) \sum_{\substack{s_{2} \in s s[r, j-1] \\
r \text { base-paired in } s_{2}}} B F\left(s_{2}\right)+ \\
& \sum_{r=i+5}^{j-5} \exp \left(-\frac{a+b}{R T}\right) \cdot \sum_{s_{1} \in s s[i+1, r-1]} B F\left(s_{1}\right) N\left(s_{1}\right) \sum_{\substack{s_{2} \in s s[r, j-1] \\
r \text { base-paired in } s_{2}}} B F\left(s_{2}\right)+ \\
& \sum_{r=i+5}^{j-5} \exp \left(-\frac{a+b}{R T}\right) \cdot \sum_{s_{1} \in s s[i+1, r-1]} B F\left(s_{1}\right) \sum_{\substack{s_{2} \in s s[r, j-1] \\
\text { base-paired in } s_{2}}} B F\left(s_{2}\right) N\left(s_{2}\right) \\
& =\exp \left(-\frac{a+b}{R T}\right) \cdot \sum_{r=i+5}^{j-5}\left[Z M_{i+1, r-1} \cdot Z M 1_{r, j-1}+\right. \\
& \left.Q M_{i+1, r-1} \cdot Z M 1_{r, j-1}+Z M_{i+1, r-1} \cdot Q M 1_{r, j-1}\right] .
\end{aligned}
$$

Now $Q B_{i, j}=A_{i, j}+B_{i, j}+C_{i, j}$. It nevertheless remains to define the recursions for $Q M 1_{i, j}$ and $Q M_{i, j}$. These satisfy the following.

$$
\begin{aligned}
Q M 1_{i, j}= & \sum_{k=i+4}^{j} \sum_{\substack{s \in s s[i, k] \\
(i, k) \in s}} \exp \left(-\frac{c(j-k)}{R T}\right) \cdot B F(s) \cdot[N(s)+\operatorname{arc1}(k+1, j)] \\
= & \sum_{k=i+4}^{j} \exp \left(-\frac{c(j-k)}{R T}\right) \cdot\left[Q B_{i, k}+Z B_{i, k} \cdot \operatorname{arc} 1(k+1, j)\right] . \\
Q M_{i, j}= & \sum_{r=i}^{j-5} \exp \left(-\frac{b+c(r-i)}{R T}\right) \cdot\left[Q M 1_{r, j}+Z M 1_{r, j} \cdot \operatorname{arc} 1(i, r-1)\right]+ \\
& \sum_{r=i}^{j-5} \exp \left(-\frac{b}{R T}\right) \cdot\left[Q M_{i, r-1} Z M 1_{r, j}+Z M_{i, r-1} Q M 1_{r, j}\right] .
\end{aligned}
$$

Suppose that $s=\left\{(i, j),\left(i_{1}, j_{1}\right), \ldots,\left(i_{k}, j_{k}\right)\right\}$ is a multiloop closed by $(i, j)$, where $i<i_{1}<j_{1}<i_{2}<j_{2}<$ $\cdots<i_{k}<j_{k}<j$. Then note that we do not count neighbors of $s$ obtained by adding a base pair $(x, y)$ to the multiloop $s$, where $i<x<i_{\ell}<j_{\ell}<y$. Due to McCaskill's trick in the treatment of multiloops in the partition function [14, the treatment of such structural neighbors appears to be impossible while retaining the run time $O\left(n^{3}\right)$. Nevertheless, multiloops are energetically costly due to entropic considerations, and so penalized in the Turner energy model. For this reason, multiloops are generally small, without many unpaired bases $x, y$ capable of forming such base pairs. If a multiloop is of sufficient size to permit such 
unpaired bases $x, y$, then the multiloop free energy is likely to be large, so when the contribution is weighted by the Boltzmann factor of $s$, the net contribution to $\langle N\rangle$ will be negligeable. 


\begin{tabular}{|cccc|}
\hline Rfam & Num Seq & EXPU & EXPB \\
\hline RF00001 & 712 & $0.367082 \pm 0.006445$ & $0.442810 \pm 0.057727$ \\
RF00004 & 208 & $0.373414 \pm 0.004549$ & $0.611431 \pm 0.116160$ \\
RF00005 & 960 & $0.373047 \pm 0.009376$ & $0.507313 \pm 0.122405$ \\
RF00008 & 84 & $0.363520 \pm 0.012268$ & $0.413163 \pm 0.085666$ \\
RF00031 & 61 & $0.369005 \pm 0.008448$ & $0.572074 \pm 0.168601$ \\
RF00045 & 66 & $0.371720 \pm 0.005207$ & $0.513964 \pm 0.068067$ \\
RF00167 & 133 & $0.364369 \pm 0.009093$ & $0.731829 \pm 0.258182$ \\
RF00375 & 130 & $0.356841 \pm 0.005719$ & $0.382784 \pm 0.058189$ \\
RF01055 & 160 & $0.368760 \pm 0.007053$ & $0.566054 \pm 0.124462$ \\
\hline
\end{tabular}

Table 1: Normalized expected number of neighbors of sequences in the seed alignment of various Rfam families [10, given as the mean plus or minus one standard deviation of values computed for each family. Column 1 contains the Rfam family name of the noncoding RNA investigated. Column 2 contains the number of sequences in the family; columns 3 and 4 respectively contain the average, taken over each Rfam family, of the expected number of neighbors of each sequence, normalized by dividing by sequence length, denoted respectively EXPU and EXPB. Computations were performed using RNAexpNumNbors with respect to the uniform probability (EXPU in column 3) and Boltzmann probability (EXPB in column 4). The table clearly shows that the Boltzmann expected number of neighbors is generally larger than the uniform expected number of neighbors.

\section{References}

[1] S.F. Altschul and B.W. Erikson. Significance of nucleotide sequence alignments: A method for random sequence permutation that preserves dinucleotide and codon usage. Mol. Biol. Evol, 2(6):526-538, 1985.

[2] G. R. Bowman and V. S. Pande. Protein folded states are kinetic hubs. Proc. Natl. Acad. Sci. U.S.A., 107(24):10890-10895, June 2010.

[3] P. Clote, F. Ferré, E. Kranakis, and D. Krizanc. Structural RNA has lower folding energy than random RNA of the same dinucleotide frequency. RNA, 11(5):578-591, 2005.

[4] R. M. Dirks, M. Lin, E. Winfree, and N. A. Pierce. Paradigms for computational nucleic acid design. Nucleic. Acids. Res., 32(4):1392-1403, 2004.

[5] Philippe Flajolet and Robert Sedgewick. Analytic Combinatorics. Cambridge University Press, 2009.

[6] C. Flamm, W. Fontana, I.L. Hofacker, and P. Schuster. RNA folding at elementary step resolution. RNA, 6:325-338, 2000.

[7] E. Freyhult, V. Moulton, and P. Clote. Boltzmann probability of RNA structural neighbors and riboswitch detection. Bioinformatics, 23(16):2054-2062, Aug 2007. doi: 10.1093/bioinformatics/btm314.

[8] E. Fusy and P. Clote. Combinatorics of locally optimal RNA secondary structures. J Math Biol., 0(O):O, December 2012.

[9] J.A. Garcia-Martin, P. Clote, and I. Dotu. RNAiFold: A constraint programming algorithm for RNA inverse folding and molecular design. Journal of Bioinformatics and Computational Biology, 11(2):1350001, 2012.

[10] P. P. Gardner, J. Daub, J. Tate, B. L. Moore, I. H. Osuch, S. Griffiths-Jones, R. D. Finn, E. P. Nawrocki, D. L. Kolbe, S. R. Eddy, and A. Bateman. Rfam: Wikipedia, clans and the "decimal" release. Nucleic. Acids. Res., 39(Database):D141-D145, January 2011.

[11] I.L. Hofacker. Vienna RNA secondary structure server. Nucleic Acids Res., 31:3429-3431, 2003.

[12] M. Huynen, R. Gutell, and D. Konings. Assessing the reliability of RNA folding using statistical mechanics. J. Mol. Biol., 267(5):1104-1112, April 1997. 


\begin{tabular}{|l|cccc|}
\hline & CMFE & EXPB & EXPU & MFE \\
\hline CMFE & 1.000000 & 0.384740 & 0.105773 & 0.327990 \\
EXPB & 0.384740 & 1.000000 & 0.028191 & 0.777432 \\
EXPU & 0.105773 & 0.028191 & 1.000000 & -0.003397 \\
MFE & 0.327990 & 0.777432 & -0.003397 & 1.000000 \\
\hline
\end{tabular}

Table 2: Pearson correlation between the length-normalized number of neighbors of the MFE structure, CMFE structure and the uniform or Boltzmann expected value. Correlations were computed over the pooled sequences from all nine Rfam families investigated. Headers are explained as follows. MFE: normalized number of neighbors for the MFE structure; CMFE: normalized number of neighbors for the CMFE structure, i.e. the structure having minimum free energy that is consistent with the Rfam consensus structure; EXPB: normalized expected number of neighbors with respect to Boltzmann probability; EXPU: normalized expected number of neighbors with respect to uniform probability. Note the complete absence of correlation between EXPB and EXPU. Results are similar when analyzing Rfam family RF00001 (5S rRNA), both normalized and unnormalized (data not shown); i.e. the lack of correlation is not due to any pooling effect of different families. Although Table 1 shows that the Boltzmann expected number of neighbors is greater than the uniform expected number of neighbors, there appears to be no correlation between these values.

\begin{tabular}{|l|ccccc|}
\hline & $\mathrm{H}$ & EnsDef & ExpBPdist & ExpNumBP & ExpNumNbors \\
\hline $\mathrm{H}$ & 1.000000 & 0.716498 & 0.699402 & -0.471001 & -0.000638 \\
EnsDef & 0.716498 & 1.000000 & 0.997092 & -0.306858 & -0.024428 \\
ExpBPdist & 0.699402 & 0.997092 & 1.000000 & -0.285362 & -0.027360 \\
ExpNumBP & -0.471001 & -0.306858 & -0.285362 & 1.000000 & -0.006972 \\
ExpNumNbors & -0.000638 & -0.024428 & -0.027360 & -0.006972 & 1.000000 \\
\hline
\end{tabular}

Table 3: Correlations of various measures that depend on the Boltzmann ensemble of all secondary structures of a given RNA sequence. All measures have been normalized by dividing by sequence length. Column and row headers are explained as follows. H: average positional entropy, EnsDef: ensemble defect to MFE structure, ExpBPdist: expected base pair distance to MFE structure, ExpNumBP: expected number of base pairs, ExpNumNbors: expected number of neighbors. See Appendix for definitions of these measures.

\begin{tabular}{|l|cccccc|}
\hline & E & MFE-EXPB & EXPB & MFE-EXPU & MFE & SeqLen \\
\hline E & 1.000000 & 0.088782 & 0.138171 & 0.135036 & 0.132890 & -0.862103 \\
MFE-EXPB & 0.088782 & 1.000000 & 0.370294 & 0.872449 & 0.872135 & -0.000543 \\
EXPB & 0.138171 & 0.370294 & 1.000000 & 0.775958 & 0.777432 & 0.125386 \\
MFE-EXPU & 0.135036 & 0.872449 & 0.775958 & 1.000000 & 0.999436 & 0.065343 \\
MFE & 0.132890 & 0.872135 & 0.777432 & 0.999436 & 1.000000 & 0.065674 \\
SeqLen & -0.862103 & -0.000543 & 0.125386 & 0.065343 & 0.065674 & 1.000000 \\
\hline
\end{tabular}

Table 4: Correlations for data pooled from all nine Rfam families. Column headers designate the following. E: minimum free energy; MFE-EXPB: (number of neighbors of MFE structure minus the Boltzmann expected number of neighbors) divided by sequence length - i.e. length-normalized; EXPB: Boltzmann expected number of neighbors, divided by sequence length; MFE-EXPU: (number of neighbors of MFE structure minus the uniform expected number of neighbors) divided by sequence length; MFE: number of neighbors of the MFE structure divided by sequence length; SeqLen: sequence length. 


\begin{tabular}{|l|cccc|}
\hline FASTA & MFE-EXP & CMFE-MFE & CMFE-EXP & BPdist \\
\hline RF00001 & $0.0293 \pm 0.0881$ & $0.2006 \pm 0.5482$ & $0.2300 \pm 0.5527$ & $0.1884 \pm 0.1614$ \\
RF00004 & $0.0543 \pm 0.1872$ & $0.2863 \pm 0.6817$ & $0.3406 \pm 0.6945$ & $0.2082 \pm 0.1243$ \\
RF00005 & $0.0447 \pm 0.1863$ & $0.4260 \pm 0.6458$ & $0.4707 \pm 0.6195$ & $0.2631 \pm 0.1265$ \\
RF00008 & $0.1336 \pm 0.0998$ & $0.0133 \pm 0.0665$ & $0.1469 \pm 0.1024$ & $0.0492 \pm 0.1143$ \\
RF00031 & $0.0772 \pm 0.3079$ & $0.1895 \pm 0.4428$ & $0.2667 \pm 0.5247$ & $0.1108 \pm 0.1207$ \\
RF00045 & $0.0364 \pm 0.1028$ & $0.5857 \pm 1.0071$ & $0.6221 \pm 0.9906$ & $0.3064 \pm 0.1882$ \\
RF00167 & $0.2412 \pm 0.3268$ & $0.0714 \pm 0.3087$ & $0.3126 \pm 0.4151$ & $0.0631 \pm 0.1175$ \\
RF00375 & $0.0344 \pm 0.1122$ & $0.0012 \pm 0.1524$ & $0.0356 \pm 0.1711$ & $0.1018 \pm 0.1455$ \\
RF01055 & $0.1148 \pm 0.1812$ & $0.2753 \pm 0.3010$ & $0.3901 \pm 0.2401$ & $0.2042 \pm 0.1400$ \\
\hline
\end{tabular}

Table 5: Table to determine whether the minimum free energy structure has more neighbors than the expected number. MFE [resp. CMFE] stands for the length-normalized number of neighbors for the minimum free energy structure [resp. the structure having minimum free energy among those structures that are consistent with the Rfam consensus structure]. EXP stands for the length-normalized expected number of neighbors, as computed by RNAexpNumNbors, and BPdist is the length-normalized base pair distance between the MFE structure and the CMFE structure. Values are given as the mean plus or minus one standard deviation, taken over all sequences in the corresponding Rfam family.

[13] D.H. Matthews, J. Sabina, M. Zuker, and D.H. Turner. Expanded sequence dependence of thermodynamic parameters improves prediction of RNA secondary structure. J. Mol. Biol., 288:911-940, 1999.

[14] J.S. McCaskill. The equilibrium partition function and base pair binding probabilities for RNA secondary structure. Biopolymers, 29:1105-1119, 1990.

[15] R. Nussinov and A. B. Jacobson. Fast algorithm for predicting the secondary structure of single stranded RNA. Proceedings of the National Academy of Sciences, USA, 77(11):6309-6313, 1980.

[16] E. Ravasz, A. L. Somera, D. A. Mongru, Z. N. Oltvai, and A. L. Barabasi. Hierarchical organization of modularity in metabolic networks. Science, 297(5586):1551-1555, August 2002.

[17] E. Senter and P. Clote. Fast, approximate kinetics of RNA folding. submitted.

[18] E. Senter, S. Sheik, I. Dotu, Y. Ponty, and P. Clote. Using the fast fourier transform to accelerate the computational search for RNA conformational switches. PLoS One, 7(12):e50506, 2012.

[19] A. Serganov, Y. R. Yuan, O. Pikovskaya, A. Polonskaia, L. Malinina, A. T. Phan, C. Hobartner, R. Micura, R. R. Breaker, and D. J. Patel. Structural basis for discriminative regulation of gene expression by adenine- and guanine-sensing mRNAs. Chem. Biol., 11(12):1729-1741, December 2004.

[20] M. Sprinzl, C. Horn, M. Brown, A. Ioudovitch, and S. Steinberg. Compilation of tRNA sequences and sequences of tRNA genes. Nucleic Acids Res., 26:148-153, 1998.

[21] P. R. Stein and M. S. Waterman. On some new sequences generalizing the Catalan and Motzkin numbers. Discrete Mathematics, 26:261-272, 1978.

[22] D. H. Turner and D. H. Mathews. NNDB: the nearest neighbor parameter database for predicting stability of nucleic acid secondary structure. Nucleic. Acids. Res., 38(Database):D280-D282, January 2010.

[23] V. Van Noort, B. Snel, and M. A. Huynen. The yeast coexpression network has a small-world, scale-free architecture and can be explained by a simple model. EMBO Rep., 5(3):280-284, March 2004.

[24] A. Wagner and D. A. Fell. The small world inside large metabolic networks. Proc. Biol. Sci., 268(1478):1803-1810, September 2001.

[25] J. Waldispuhl and P. Clote. Computing the partition function and sampling for saturated secondary structures of RNA, with respect to the Turner energy model. J. Comput. Biol., 14(2):190-215, March 2007. 
[26] D. J. Watts and S. H. Strogatz. Collective dynamics of 'small-world' networks. Nature, 393(6684):440442, June 1998.

[27] S. Wuchty. Small worlds in RNA structures. Nucleic. Acids. Res., 31(3):1108-1117, February 2003.

[28] S. Wuchty, W. Fontana, I.L. Hofacker, and P. Schuster. Complete suboptimal folding of RNA and the stability of secondary structures. Biopolymers, 49:145-164, 1999.

[29] T. Xia, Jr. J. SantaLucia, M.E. Burkard, R. Kierzek, S.J. Schroeder, X. Jiao, C. Cox, and D.H. Turner. Thermodynamic parameters for an expanded nearest-neighbor model for formation of RNA duplexes with Watson-Crick base pairs. Biochemistry, 37:14719-35, 1999.

[30] M. Zuker, D. H. Mathews, and D. H. Turner. Algorithms and thermodynamics for RNA secondary structure prediction: A practical guide. In J. Barciszewski and B.F.C. Clark, editors, RNA Biochemistry and Biotechnology, NATO ASI Series, pages 11-43. Kluwer Academic Publishers, 1999. 